\title{
Breast Cancer Arising from a Suspected Fibroadenoma during Pregnancy: A Case Report and Review of Literature
} Yasamin Ghazvini Kor*, Mehdi Saeedan and Mohamed Sobhy Badr Sobei

Surgery Department, Thumbay Hospital, Ajman, Dubai, UAE

\begin{abstract}
Introduction: Fibroadenomas are the most common benign neoplastic lesion of the breast. These hormonesensitive tumors can grow rapidly under the influence of pregnancy hormones. Although rare, malignant transformation of these lesions has been reported. The risk of the standard treatment regimen on the fetus makes the PABC (Pregnancy-Associated Breast Cancer) a challenging task for clinicians. Overall treatment is based on guidelines for the general population with some variations to decrease the risk of fetal damage as much as possible.
\end{abstract}

Case report: A 37-year-old Indian female, presented with a palpable mass in her right breast in the first month of pregnancy. A preliminary diagnosis of fibroadenoma was made at the time. She came back at 39 weeks of gestation with severe vaginal bleeding and a huge painful mass in the right breast. Labor was induced upon diagnosis of intrauterine fetal death and ultrasonography of the breast revealed a lobulated soft tissue mass with multiple cystic areas and internal vascularity; measuring $16 \mathrm{~cm}$ in largest diameter. Excisional biopsy under GA (General Anesthesia) was performed upon request of the patient and microscopic HPE (Histopathological Examination) reported the presence of Grade 3 invasive ductal carcinoma.

Conclusion: Breast cancer arising within fibroadenoma during pregnancy is extremely rare but presents unique challenges in evaluation and management. An enlarging breast mass during pregnancy should never be neglected, and thorough investigation including biopsy is strongly advised to establish a definitive diagnosis.

Keywords: Breast cancer; Pregnancy-associated breast cancer; Fibroadenoma; Malignant transformation

\section{Introduction}

All breast lesions seen in the population may develop during pregnancy. Most common problems fall within a spectrum of infectious conditions followed by fibroadenoma and finally breast cancer [1]. Fibroadenoma is considered the most common neoplastic lesion of the breast in both non-pregnant and pregnant populations. This hormone-sensitive tumor is usually observed in early reproductive life, and high level of hormones during pregnancy may provide a favorable environment for this lesion to grow sometimes out of proportion $[2,3]$. Transformation of fibroadenoma to a malignant lesion is extremely rare, but nevertheless, it is still a possibility and has been reported in several studies to be around $0.02 \%$ to $0.1 \%$ [4-6]. Breast cancer which occurs during pregnancy, in the first postpartum year or anytime during lactation is identified as PABC (pregnancy-associated breast cancer) and presents a challenging clinical situation in diagnosis and treatment [7]. Hormonal and physiological changes within the breast tissue may impede the accurate interpretation and analysis of clinical examinations of the breasts. Since even a short delay in the timely diagnosis and proper management may lead to the transformation of a benign fibroadenoma into a breast cancer, any breast mass discovered during pregnancy should be investigated carefully [8]. Pregnancyassociated breast cancer treatment is always a clinical challenge due to the worrisome risk of the standard treatment regimen on the fetus in on hand and danger of suboptimal treatment to the mother in another hand [9]. When PABC is diagnosed, surgery is always the mainstay treatment and based on clinical circumstances either a mastectomy or breastconserving surgery may be considered. Other treatment modalities like radiotherapy and chemotherapy though less favorable, may be used when indicated and after thorough evaluation and assessment [10].

\section{Case Report}

A 37-year-old Indian female, G6 P4 A1 presented to OBG department with a palpable mass in her right breast in the first month of pregnancy. The patient was a known case of uncontrolled diabetes mellitus for six years and asthma for 12 years and currently on insulin and $\beta 2$ agonist. She had a history of laparotomy six years ago for an unknown abdominal cyst and a salpingectomy of the right fallopian tube four years ago. There was no family history of breast cancer. She experienced menarche at the age of 12 with regular periods since then, she got married at the age of 18 , and her first pregnancy was at the age of 19; she had four live birth, and all of the babies were breastfed for at least one year. There was no history of oral contraceptive use. Ultrasonography at the time of admission found a $2.4 \mathrm{~cm} \times 2 \mathrm{~cm}$ mass and the soft tissue nodules along the upper outer quadrant of right breast. A preliminary diagnosis of fibroadenoma with cystic changes was made, but despite the strict advice of obstetrician, patient refused to do FNAC (Fine Needle Aspiration Cytology) and further evaluation at the time. The course of pregnancy was uneventful until week 39 of gestation when the patient presented with severe vaginal bleeding as well as a noticeably large mass in her right breast, labor was induced upon diagnosis of intrauterine fetal death, and the patient was referred to the surgery department for further evaluation of her breast mass. According to the patient, the mass has been progressively increasing in size during pregnancy and has been painful in last two months. There was not any history of systemic symptoms. On physical evaluation,

*Corresponding author: Yasamin Ghazvini Kor, Surgery Department, Thumbay Hospital, Ajman, Dubai, UAE, Tel: 00971558117437; E-mail: jasmin.ghazvini@ gmail.com

Received June 25, 2017; Accepted August 04, 2017; Published August 10, 2017

Citation: Kor YG, Saeedan M, Badr Sobei MS (2017) Breast Cancer Arising from a Suspected Fibroadenoma during Pregnancy: A Case Report and Review of Literature. Cancer Sci Ther 9: 589-592. doi: 10.4172/1948-5956.1000478

Copyright: @ 2017 Kor YG, et al. This is an open-access article distributed under the terms of the Creative Commons Attribution License, which permits unrestricted use, distribution, and reproduction in any medium, provided the original author and source are credited. 
patient's BMI was 25 , and vital signs were within normal limits. On local examination of the right breast, there was an immobile, hard huge mass measuring about $16 \mathrm{~cm}$ with a lobular surface occupying whole right breast. There was no skin change, ulceration, nipple discharge or axillary lymph node enlargement. Ultrasonography at this time revealed a well-defined lobulated soft tissue mass with multiple cystic areas and internal vascularity; measuring $15.8 \mathrm{~cm} \times 12.7 \mathrm{~cm} \times 5.8 \mathrm{~cm}$, located on the outer half of the right breast which compressed rest of the breast tissue. There was another similar lesion at breast tail measuring $9.7 \mathrm{~cm} \times 5.8 \mathrm{~cm}$. Marked subcutaneous edema underlying the lesion was also noted. The surgeon proposed a tru-cut needle biopsy as the next proper diagnostic procedure, but the patient refused and decided to undergo excisional biopsy under general anesthesia. In the operation theater, whole mass was excised in toto and sent for histopathological evaluation. Figure 1 Microscopic HPE reported the presence of Grade 3 invasive ductal carcinoma with Indefinite lymphatic infiltration Figure 2. The patient refused any further assessment and treatment and decided to continue the treatment in her home country.

\section{Discussion}

During pregnancy, a woman's breasts undergo several physiological changes. These are hyperplastic changes which occur due to elevated levels of estrogen and progesterone. In particular, under the influence of estrogen, ductal proliferation accelerates which in turn causes a significant growth of vasculature accompanied by increased blood flow and infiltration of mononuclear cells. These changes are believed to provide the proper milieu for neoplastic lesions during pregnancy $[10,11]$. Fibroadenoma is the most commonly detected neoplastic lesion during pregnancy. It can be a preexisting lesion which increases in size under the effect of pregnancy hormones or may appear de novo; in rare cases, especially in giant fibroadenomas infarction inside the lesion may occur [12]. These benign tumors of the breast, commonly found in young women between the ages of 15 and 35 years, generally present as multiple painless, firm and mobile nodules which can be managed conservatively $[2,3]$. While fibroadenomas are usually small but rapid growth has been observed in $0.5 \%$ to $2 \%$ of cases $[2,4,13]$. Although fibroadenomas are not considered premalignant, rarely breast

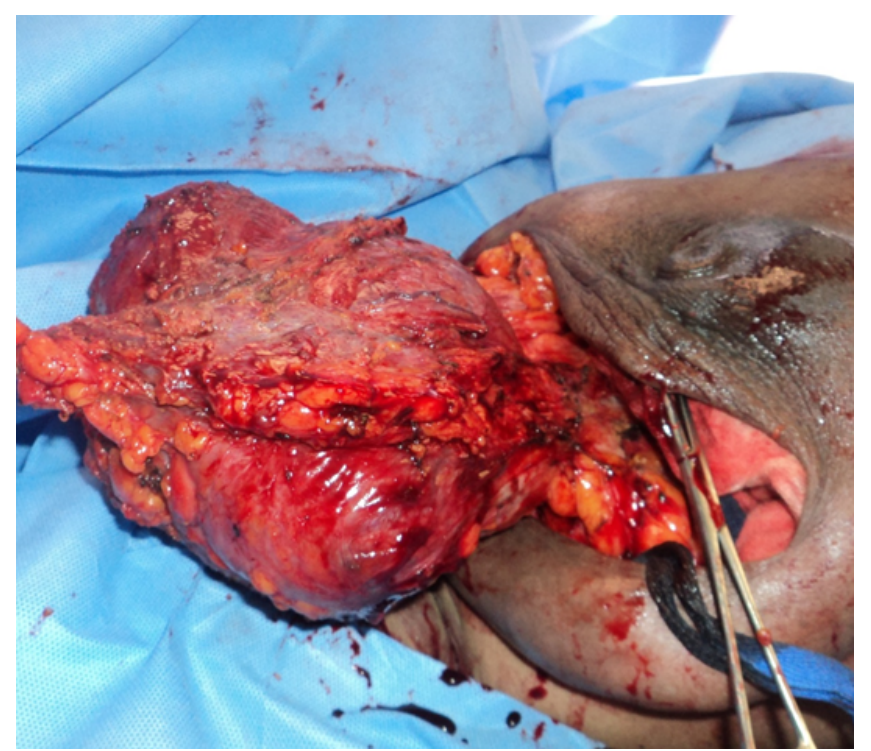

Figure 1: In toto excised right breast mass, measuring $16 \mathrm{~cm} \times 14 \mathrm{~cm} \times 8$ $\mathrm{cm}$.

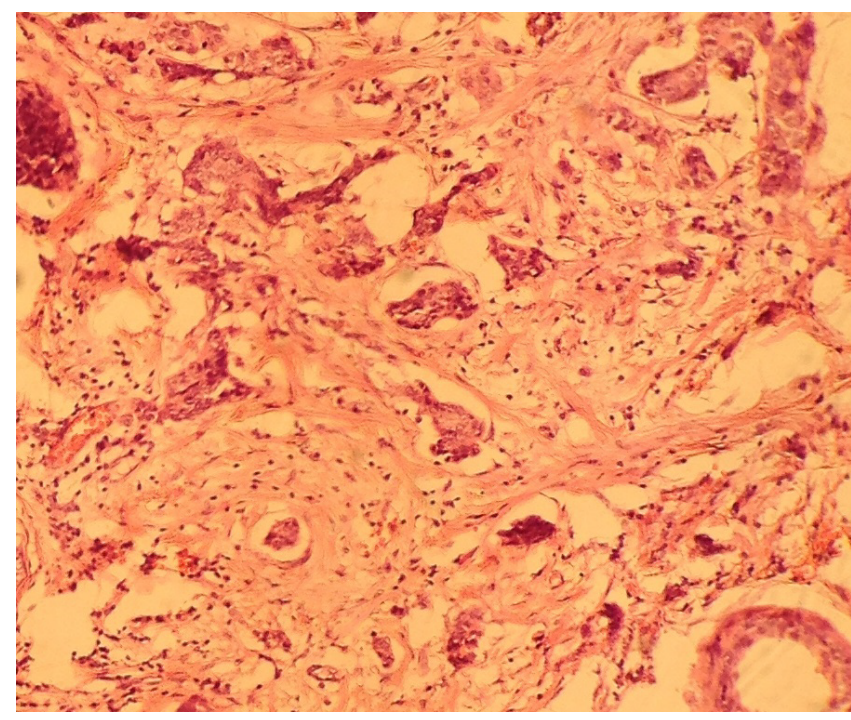

Figure 2: Poorly differentiated ductal carcinoma infiltrating the stroma obtained from a biopsy of the right breast.

cancer may arise within these lesions. Occasional cases of malignancy arising from fibroadenomas have been reported in women between the ages of 41 and 44 years $[5,14,15]$. In the literature, the incidence of malignancy arose from fibroadenoma ranges from $0.02 \%$ to $0.1 \%$ [16]. The exact frequency of this transformation is nearly impossible to determine as some larger carcinomas may replace a fibroadenoma in the course of malignant growth [15]. However, when such transformation occurs, carcinomas in situ is the most commonly found lesion with the incidence of $70 \%$ to $95 \%$; invasive carcinoma which occurs less frequently is reported to be present in $5 \%$ to $30 \%$ of cases $[4,5,16,17]$. Although extremely rare, sarcomas such as angiosarcoma, osteosarcoma have also been reported to arise within fibroadenomas $[18,19]$. There is not much data about the malignant transformation of fibroadenoma during pregnancy, researchers have not been able to propose the exact molecular mechanism which initiates and propagates the progression of malignancy within fibroadenoma, but the rapid growth of fibroadenoma under the effect of pregnancy hormones may play an important role [20,21]. Although comparative studies in determining the natural history of fibroadenomas seem to be equivocal, Kuijper et al. in a clonality study showed that given the right condition fibroadenomas could progress in both epithelial (carcinoma) and stromal direction (phyllodes tumor) [22]. Higher incidence of CIS (Carcinoma in situ) found in transforming fibroadenoma highlights the importance of appropriate evaluation of such condition because, by early diagnosis and proper management, breast CIS is highly manageable with an excellent prognosis [23]. Breast cancer is the most common malignancy occurring during pregnancy. It may complicate 1 in 3000 pregnancies $[1,2,7,24,25]$. The tendency of women in delaying the pregnancy is expected to increase the incidence of this problem in future [24,25]. The diagnosis of breast cancer in pregnant women follows the same principles as in non-pregnant cases. It is based on an assessment of clinical examination, imaging, and biopsy. Any breast mass during pregnancy should be viewed as a potential malignancy, and systematic investigation must be done if the mass persists for more than two weeks. There are considerable challenges in the diagnosis and management of breast cancer during pregnancy. Physiological changes in the breast tissue and the risk of radiation exposure to the fetus may hold back the necessary clinical evaluation [7]. Another challenge in 
the management of breast cancer during pregnancy arise from the fact that pregnancy associated immunological and hormonal changes may augment the growth of cancerous cells more intensely; hence even a short delay of just 1-2 months in the start of therapy may change the outcome greatly $[25,26]$. Despite the importance of precise timing, the delay in the diagnosis of pregnancy-associated breast cancer has been reported to be common and is believed to be the cause of presentation of the patients in the more advanced stages of the disease and consequently with a poorer prognosis. These delays do not happen only because of the difficulty in the interpretation of clinical investigations, but sometimes also the unwillingness of the physician to do a biopsy during pregnancy plays a role [27]. Similar to the general population, genetic predisposition may increase the risk of breast cancer in pregnant women as well, it is postulated that in women with a mutation in BRCA2, the protective effect of multiparity on breast cancer has been lost $[9,28$ 30]. Family history as a strong risk factor may increase the possibility of breast cancer in young women by at least two-fold. However, studies fail to show any significant increase in the cancer risk among pregnant women with the family history of breast cancer when compared to women without a family history $[31,32]$. Similar to the general population the most commonly found subtype of breast cancer during pregnancy is infiltrating ductal carcinoma. However, breast cancers during pregnancy tend to be poorly differentiated high-grade lesions at the time of diagnosis. Additionally, inflammatory breast cancer has been reported to be more frequently seen in pregnant women although the literature is not consistent about it [33-36]. Although well-being of the fetus is a factor in the management of pregnancy-associated breast cancer, it is important to understand that overall treatment is based on guidelines for the general population with some changes to decrease the risk of fetal damage as much as possible. Since any delay in initiation of the treatment may have grave consequences, the clinicians must approach these cases with high vigilance and curative intent [7]. The decision to continue the pregnancy is made based on clinical evaluation and staging of the disease in coordination with patient preference; when such a decision is made, mastectomy remains the treatment of choice. Harmful effects of radiotherapy on fetus make it inadvisable during the first and second trimesters, so breast conserving surgery (a lumpectomy and a partial mastectomy plus radiation therapy) may be considered only in the third trimester [37]. Sentinel node biopsy also utilizes the radiation as part of the procedure so axillary lymph node dissection should be used for surgical staging [25,38]. Teratogenic effects of chemotherapeutic agents are believed to be limited to the period of organogenesis, so the use of this treatment modality is relatively safe after the first trimester [39]. Although hormonal therapy is a promising adjuvant therapy in non-pregnant receptor positive patients, its use during pregnancy is not recommended [25,40]. Despite the previous belief, clinical studies demonstrate that termination of pregnancy would not improve the survival. Hence it is not advised anymore, but if breast cancer is diagnosed in the early stage of gestation, cessation of pregnancy may become a more viable choice considering the risks associated with a delay in standard treatment [39]. In general, the fiveyear survival rate in PABC is similar to the non-pregnant population; however, two primary risk factors which may affect the survival rate of $\mathrm{PABC}$ are a history of recent pregnancy and delay in treatment for 3 to 6 months [41-44].

\section{Conclusion}

Breast cancer arising within fibroadenoma during pregnancy is extremely rare but presents unique challenges in evaluation and management. An enlarging breast mass during pregnancy should never be neglected, and thorough investigation including biopsy is strongly advised to establish a definitive diagnosis. It is vital to have a multidisciplinary approach toward diagnosed PABC in which well-being of both mother and fetus is considered carefully. Higher incidence of CIS found in transforming fibroadenoma necessitates the judicious evaluation of such condition because, by early diagnosis and proper management, breast CIS is highly manageable with an excellent prognosis.

\section{Acknowledgment}

We would like to express our sincere gratitude to our colleagues in the departments of surgery and OB/GYN in Thumbay hospital, Ajman who provided insight and expertise that greatly assisted this paper.

\section{Disclosure of Interests}

The authors of this manuscript declare no conflicts of interest in the authorship or publication of this contribution.

\section{Contribution to Authorship}

The authors of this paper certify that they have participated sufficiently in the work to take public responsibility for the entire content of the manuscript.

\section{Funding Disclosures}

The authors of this paper certify that no funding has been received for the conduct of this study and/or preparation of this manuscript.

\section{Ethics Approval}

This article does not contain any studies with animals performed by any of the authors. Informed consent was obtained from the patient and the study was approved by the Ethics Committee at Thumbay Hospital, Ajman, UAE.

\section{References}

1. Cacala S (2010) Breast conditions during pregnancy and lactation. CME 28 508

2. Scott-Conner CEH, Schorr SJ (1995) The diagnosis and management of breas problems during pregnancy and lactation. Am J Surg 170: 401-405.

3. Hughes LE, Mansel RE, Webster DJT (1989) Breast anatomy and physiology, In: Hughes LE, Manset RE, Webster DJT (eds): Benign disorders and diseases of the breast: Concepts and clinical management. London, Balliere Tindall 5-13.

4. El Wakeel H, Umpleby HC (2003) Systematic review of fibroadenoma as a risk factor for breast cancer. Breas 12: 302-307.

5. Diaz NM, Palmer JO, McDivitt RW (1991) Carcinoma arising within fibroadenomas of the breast. A clinicopathologic study of 105 patients. Am J Clin Pathol 95: 614-622.

6. Abe M, Miyata S, Nishimura S, Lijima K, Makita M, et al. (2011) Malignant transformation of breast fibroadenoma to malignant phyllodes tumor: long-term outcome of 36 malignant phyllodes tumors. Breast Cancer 18: 268-272.

7. Litton JK (2001) Gestational breast cancer: Epidemiology and diagnosis. In Up-to-date. Post.

8. Psyrri A, Burtness B (2005) Pregnancy-associated breast cancer. The Cancer Journal 11: 83-95.

9. Ishida T (1992) Clinicopathologic characteristics and prognosis of breast cancer patients associated with pregnancy and lactation: Analysis of case control study in Japan. Jpn J Cancer Res 83: 1143-1149.

10. Rosen PP (2001) Anatomic and physiologic morphology. In: Rosen PP, (ed) Rosen's breast pathology. (2nd edn), Lippincott-Raven, Philadelphia (PA), USA. pp. 1-21.

11. Leveno K, Cunningham F, Alexander J, Bloom S, Casey B, et al. (2007) Williams manual of obstetrics: Pregnancy complications. McGraw Hill Professional USA.

12. Langer A, Mohallem M, Berment H, Ferreira F, Gog A, et al. (2015) Breast lumps in pregnant women. Diagn Interv Imaging 96: 1077-1087.

13. Rosen PP (2001) Fibroepithelial neoplasms. In: Rosen PP, (ed). Rosen's breast pathology. (2nd edn), Philadelphia, USA. pp. 163-200.

14. Fukuda M, Nagao K, Nishimura R, Matsuda M, Baba K (1989) Carcinoma arising in fibroadenoma of the breast-a case report and review of the literature. Jpn J Surg 19: 593-596. 
Citation: Kor YG, Saeedan M, Badr Sobei MS (2017) Breast Cancer Arising from a Suspected Fibroadenoma during Pregnancy: A Case Report and Review of Literature. Cancer Sci Ther 9: 589-592. doi: 10.4172/1948-5956.1000478

15. Konakry BK, Harrison EG, Payne WS (1975) Lobular carcinoma arising in fibroadenoma of the breast. Cancer 35: 450-456.

16. Pick PW, Lossifides IA (1984) Occurrence of breast carcinomas within a fibroadenoma: A review. Arch Pathol Lab Med 108: 590-594.

17. Oyyello L, Gump FE (1985) The management of patients with carcinomas in fibroadenomatous tumors of the breast. Surg Gynecol Obstet 160: 99-104.

18. Babarovic E, Zamolo G, Mustac E, Strcic M (2011) High grade angiosarcoma arising in fibroadenoma. Diagn Pathol 6: 125.

19. Killick SB, McCann BG (1995) Osteosarcoma of the breast associated with fibroadenoma. Clin Oncol 7: 132-133.

20. Park CA, David LR, Argenta LC (2006) Breast asymmetry: Presentation of a giant fibroadenoma. Breast J 12: 451-461.

21. Greydanus DE, Parks DS, Farrell EG (1989) Breast disorders in children and adolescents. Pediatr Clin North Am 36: 601-638.

22. Kuijper A, Buerger H, Simon R, Schaefer KL, Croonen A, et al. (2002) Analysis of the progression of fibroepithelial tumors of the breast by PCR-based clonality assay. J Pathol 197: 575-581.

23. Subhedar P, Olcese C, Patil S, Morrow M, Van Zee KJ (2015) Decreasing recurrence rates for ductal carcinoma in situ: Analysis of 2996 women treated with breast-conserving surgery over 30 years. Ann Surg Oncol 22: 3273-3281.

24. Van Calsteren K, Heyns L, De Smet F, Van Eycken L, Gziri MM, et al. (2010) Cancer during pregnancy: An analysis of 215 patients emphasizing the obstetrical and the neonatal outcomes. J Clin Oncol 28: 683-689.

25. Loibl S, Minckwitz Gv, Gwyn K, Ellis P, Blohmer JU, et al. (2006) Breast carcinoma during pregnancy. Cancer 106: 237-246.

26. Gentilinia O, Masullo M, Rotmensz N, Peccatori F, Mazzarol G, et al. (2005) Breast cancer diagnosed during pregnancy and lactation: Biological features and treatment options. EJSO 31: 232-236.

27. Petrek J (1991) Pregnancy-associated breast cancer. Seminars in Surgical Oncology 7: 306-310.

28. Shen T, Vortmeyer AO, Zhuang Z, Tavassoli FA (1999) High frequency of allelic loss of BRCA2 gene in pregnancy-associated breast carcinoma. J Natl Cancer Inst 91: 1686-1687.

29. Johannsson O, Loman N, Borg A, Olsson H (1998) Pregnancy-associated breast cancer in BRCA1 and BRCA2 germline mutation carriers. Lancet 352 : 1359-1360.

30. Cullinane CA, Lubinski J, Neuhausen SL, Ghadirian P, Lynch HT, et al. (2005) Effect of pregnancy as a risk factor for breast cancer in BRCA1/BRCA2 mutation carriers. Int J Cancer 117: 988-991.
31. Pharoah PD, Day NE, Duffy S, Easton DF, Ponder BA (1997) Family history and the risk of breast cancer: A systematic review and meta-analysis. Int $\mathrm{J}$ Cancer 71: $800-809$

32. Johansson AL, Andersson TM, Hseih CC, Cnattingius S, Dickman PW, et al. (2015) Family history and risk of pregnancy-associated breast cancer (PABC). Breast Cancer Res Treat 151: 209-217.

33. Anderson BO, Petrek JA, Byrd DR, Senie RT, Borgen PL (1996) Pregnancy influences breast cancer stage at diagnosis in women 30 years of age and younger. Ann Surg Oncol 3: 204-211.

34. Stensheim H, Møller B, Van Dijk T, Fosså SD (2009) Cause-specific survival for women diagnosed with cancer during pregnancy or lactation: A registry-based cohort study. J Clin Oncol 27: 45-51.

35. Middleton LP, Amin M, Gwyn K, Theriault R, Sahin A (2003) Breast carcinoma in pregnant women: Assessment of clinicopathologic and immunohistochemical features. Cancer 98: 1055-1060.

36. Reed W, Hannisdal E, Skovlund E, Thoresen S, Lilleng P, et al. (2003) Pregnancy and breast cancer: A population-based study. Virchows Arch 443: 44-50.

37. National Cancer Institute (2004) Breast Cancer and Pregnancy. In: Cancer Topics. National Cancer Institute.

38. Woo JC, Yu T, Hurd TC (2003) Breast cancer in pregnancy. Arch Surg 138 91-98.

39. Eedarapalli P, Jain S (2006) Breast cancer in pregnancy. J Obstet Gynecol 26: $1-4$.

40. Ring AE, Smith IE, Ellis PA (2005) Breast cancer and pregnancy. Ann Oncol 16: $1855-1860$.

41. Amant F, Deckers S, Calsteren KV, Loibl S, Halaska M, et al. (2010) Breast cancer in pregnancy: Recommendations of an international consensus meeting. Eur J Cancer 46: 3158-3168.

42. Litton JK, Warneke CL, Hahn KM, Palla SL, Kuerer HM, et al. (2013) Case control study of women treated with chemotherapy for breast cancer during pregnancy as compared with nonpregnant patients with breast cancer. Oncologist 18: 369-376.

43. Azim HA Jr, Santoro L, Russell-Edu W (2012) Prognosis of pregnancyassociated breast cancer: a meta-analysis of 30 studies. Cancer Treat Rev 38: 834-842

44. Yang Y, Chan K, Hsieh F, Chang L, Wang M (2014) Pregnancy-associated breast cancer in Taiwanese women: Potential treatment delay and impact on survival. PLoS One 9: e111934. 